\title{
CANTABA Y CANTÉ. SOBRE UNA HIPÓTESIS TEMPORAL Y ALGUNA DE SUS REPERCUSIONES
}

\author{
ALEXANDRE VEIGA \\ Universidade de Santiago de Compostela \\ Campus de Lugo
}

0. Sobre la tcoría temporal que hemos defendida y aplicado en tantas ocasiones ${ }^{1}$ hemos vuelto en un estudio muy recientemente publicado (Veiga 2004), en que procedimos al análisis de los valores gramaticales expresados en el sistema verbal español por la forma cantab $a^{2}$. Dicho estudio ha visto la luz en el marco de una antología donde, en directo contraste con nuestra concepción de la estructura modo-temporal del verbo español, predominan con mucho las defensas de una caracterización aspectual para el valor gramatical del «imperfecto» (sin ir más lejos, la idea defendida por García Fernández 1998, 1999, 2004) y son recurrentes las alusiones al modelo interpretativo de Reichenbach (1947).

1. A propósito de este modelo interpretativo, queremos comenzar manifestando una cierta extrañeza hacia la devoción que parecen profesarle algunos de sus seguidores en el mundo de la lingüística hispánica, máxime si tenemos en cuenta que, sin pretender, ni muchísimo menos, restar valor a una teoría con alguno de cuyos fundamentos estamos suficientemente de acuerdo, lo cierto es que, precisamentc cn cl ámbito hispano, su posible aportación no supuso grandes novedades frente al modelo que ya más de un siglo antes hab́́a elaborado Andrés Bello (1841); antes bien, frente a dicho modelo ofrecía ciertas insuficiencias que ya en su día llevaron a Comrie (1981, 1985: cap. 6) a una reelaboración del sistema reichenbachiano de representaciones temporales, precisamente para obtener, mutatis mutandis, un esquema similar cn lo básico al propugnado por Bello.

\footnotetext{
Cfr., sobre todo, Veiga (1991: cap. IV) y los diferentes trabajos recogidos en (Veiga 2002).

2 Nuestra participación en el curso El verbo y sus clasificaciones (programa europeo 28588-IC-5-2000-1-ESERASMUS-EPS-1), organizado en la Universidad de Alicante (curso 2001-02) por el Catedrático Dr. D. José Luis Cifuentes Honrubia, a quien queremos agradecer muy sincerannente su invitación a que participásemos, consistió en la pronanciación, en concreto el 13 de marzo de 2002 , de una conferencia titulada «I a base gramatical de la oposición canté / cantabà. Dicha conferencia sirvió de primera y parcial presentación de un trabajo que, titulado definitivamente «La forma verbal cantaba y la estructura modo-temporal del verbo español», ha visto la luz en un volumen que reúne una serie de aportaciones en torno al estudio del «pretérito imperfectos (García Fernández \& Camus Bergareche 2004). Hemos querido aprovechar cl amable ofrecimiento del Dr. Cifientes Honrubia a contribuir, en cualquier caso, y pese a haber sido ya publicado el trabajo que sirvió de basc a aquclla conferencia, al presente volumen con este mucho más modesto escrito en que efectuamos algunas reflexiones a raíz de la aparición del moncionado tomo colectivo, en concreto a propósito de algunas ideas recogidas en su estudio introductorio (García Fernández 2004) y de la hipótesis tcmporal en él aplicada (no olvidando expresar igualmente nuestro sincero agradecimiento al Dr. García Fernández, que en su día nos invitó a participar en dicho tomo).
} 
Tal vez el relativo auge de ciertas corrientes de pensamiento que, revelando una vez más las tendencias al movimiento pendular en el devenir de la investigación lingǘstica y acaso por un cierto hastío tras muchas décadas de invocación de ciertos principios del estructuralismo, se han vuelto contra todo lo que suene (al menos contra todo lo que suene demasiado) a lingüística estructural o funcional, en exagerada reacción que parece querer negar a la Lingüística su autonomía como disciplina para volver a planteamientos que pudimos considerar superados, haya propiciado la tardía divulgación entre nosotros —desde hace, redondeando, unos tres lustros en el marco de la universidad española ${ }^{3}$ - de un estudio de base lógica publicado en el año 1947 por un autor consciente de que su análisis se basaba en una reflexión en términos de symbolic logic y podía no coincidir realmente con los hechos de estructura temporal visibles en una lengua concreta (cfr. Reichenbach 1947: 298).

$\mathrm{Y}$, desde luego, nos permitimos suponer que esa aureola (tantas veces puro espejismo) que todo lo publicado en lengua inglesa posee en los ámbitos científicos del mundo hispano (y lo mismo podríamos aplicar a otros «mundos») haya tenido algo que ver en la sobrevaloración de que la teoría de Reichenbach está siendo objeto entre nosotros, pues es una triste realidad que lo que debiera ser expediente práctico ante la difusión alcanzada por una lengua en la comunidad científica internacional ha llegado a convertirse en irreflexiva y acomplejada actitud hacia todo lo que nos llega a través de dicha lengua, con el resultado del igualmente irreflexivo y acomplejado desprecio de lo producido en alguna de las lenguas propias que en tantas ocasiones se aprecia en resultados como que, por ejemplo, se publiquen trabajos sobre lengua española con su repertorio de referencias bibliográficas poblado de títulos en inglés al mismo tiempo que se llega a silenciar la obra de bien importantes estudiosos que profesaron en nuestro país o en alguna nación lingüísticamente hermana y que, infelices ellos, publicaron sus trabajos en castellano.

Mas hemos de ser justos y no pretender que una tan somera generalización, con su punto de indignación, pueda hacer recaer acusaciones indiscriminadamente, máxime tenicndo cn cuenta que nos disponemos a comentar opiniones concretas de algún autor concreto.

1.1. Hemos de puntualizar, no obstante, que nos permitimos hablar de sobrevaloración de la referida teoría ante una afirmación como, por cjemplo, la de que «se revela como una hipótesis superior a la de los modelos en que el tiempo gramatical puede ser expresión de la relación entre un número variable de puntos» (García Fernández 1999: § 4).

Tal afirmación, que supone un rechazo hacia modelos representativos como los de $\mathrm{Be}$ llo, Rojo o, ya no digamos, como el nuestro ${ }^{4}$, que da cuenta del posible encadenamiento

3 Tomemos el trabajo de Acero (1990) como punto de referencia en este sentido, aun cuando la importancia de Comrie (1985) tenga que ser tenida en consideración dentro y fucra del ámbito de la investigación hispana. Poca difusión pudo tener, en cambio, el estudio de Baquero \& Pardo (1981), contemporáneo de la revisión a que Comrie (1981) sometió el sistema de Reicherbach (1947), que supuso ya entonces la aplicación de una teoría de «relaciones enlre puntos» al verbo español, con imporiantes diferencias frente a esta última hipótesis, como la admisión de formulaciones temporales con más o menos de los tres puntos por ella exigidos y la consideración independiente de las oricntaciones de anterioridad y posterioridad, imposible de acucrdo con los esquemas reichenbachianos (cfr. infra \$ 1.1).

4 Cfr. las contraposiciones directas que el mismo autor establece entre los postulados de Reicheribach y los nuestros (junto con los de Bello, Buil y Rojo) en (García Fernández 2004: § 2). Precisemos respeclo de Bull (1960) que este autor concibió un número potenciamente intinito de «ejes de orientación), pero exponiendo que no suele pasar de cuatro, si es que en alguna lengua llega a supcrar csta cifra (cft. Bull 1960: 23) y presentando, por medio de la combinación entre los antedichos cuatro ejes temporales y cada una de las tres orientaciones vectoriales de anterioridad, simultaneidad y posterioridad, un hipotético sistema temporal con doce posibilidades como máximo (cfr. loc. cit.). 
potencialmente infinito de orientaciones temporales entre puntos diversos (cfr. infra $\S 1.2$ ), en favor de un csquema considerablemente más rígido que, desde su propia concepción, enmascara o distorsiona ciertos hechos.

Por ejemplo, y para comcnzar, su reducción, en las fórmulas empleadas para representar las significaciones de los tenses, de las relaciones temporales a, de hecho, simultaneidad y sucesividad, desconsiderando la formalización independiente de las relaciones de anterioridad y posterioridad, supone una desventaja cuya primera consecuencia es la movilidad que de unas a otras fórmulas ofrece el punto $S$ (= point of speech, Reichenbach 1947: 288, 290) frente a su concepción como centro de relaciones temporales («The tenses detcrmine time with reference to the time point of the act of speech», 1947: 287-8; «We choose the point of speech as the starting poini», 1947: 296). Así, por ejemplo, ante formulacioncs como $E-R-S, S-E-R$ o $E-S-R$ (cfr. 1947: 297), respectivamente entendidas como epoint of event anterior al point of reference, a su vez anterior al point of speech), upoint of speech anterior al point of event, a su vez anterior al point of references y upoint of event anterior al point of speech, a su vez anterior al point of references, vemos quc cl sistcma de representaciones elegido no puede señalar de ninguna manera la posición central que a dicho punto se ha asignado desde el comienzo en esta teoría.

La razón se halla en la raíz lógica (en los postulados de «the schema which we try to construct in symbolic logic», 1947: 298) de las propias descripciones. Reichenbach (1947: $290 \mathrm{ss}$ ) representó el fluir temporal (del time, no, obviamente, del tense) en forma de vector orientado de izquierda a derecha (en el sentido, pucs, del avance de la escritura en alfabeto latino) en el cual se señalan los diferentes puntos, de tal manera que, considerando dos cualesquiera de ellos, solamente resultan posibles dos interrelaciones: la que de hecho reúne anterioridad y posterioridad (anterioridad del punto representado más a la izquierda respecto del situado más a la derccha y, consecuentemente, posterioridad de este último respecto de aquel $^{5}$ y la de simultaneidad, simbolizada por una coma situada entre las representaciones de los dos puntos temporalmente coincidentes ${ }^{6}$; por ejemplo, la siguiente figura representa el esquema temporal atribuido al Simple Past (ej. del autor: I saw John, 1947: 290):

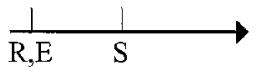

5 Notcmos que Reichenbach comenzó considerando la existencia de tres orientaciones temporales, en concreto al exponer que «Then the three indications, 'before the point of speech', 'simultaneous with the point of speech', and 'after the point of speech', furnish only three tenses» (1947: 288) y que más adelante volvería a referirse a las mismas tres posibilidades, tanto en el caso de $R$ respecto de $S$ como en el de $E$ respecto de $R$ (1947: 296). Pero la adopción de un sistema de representaciones basado en una concepción unidireccional del «tiempo) (time) le impediría individualizar recíprocamente la anterioridad y la posterioridad. No resultará ocioso recordar la afirmación de Benveniste (1965: $\S 5$ ) en el sentido de que sla langue conceptualise le temps toul autrement que ne le fait la réflexiony y reiterar la inaplicabilidad a conceptos gramaticales de concepciones derivadas de la consideración del tiempo en los sentidos físico o filosófico del término (cfr. Rojo 1974: $\$ 1.2$ ).

6 En la presentación de esta segunda interrelación el autor dice textualmente que sIn some tenses, two of the three points are simultaneous» (1947: 289). Con referencia al significado del Present Perfect dirá que «In the statement 'I have seen Charles' the event is also before the point of speech, but it is referred to a point simultaneous with the point of speech; i.e., the points of speech and reference coincides (ibid.). Obscrvemos y destaquemos que el uso de la expresión coincide se refiere estrictamente a una relación de simultaneidad (coincidencia en la línea temporal) entre dos puntos diferentes, nunca a la identificación de ambos puntos como uno solo, pues todos los esquemas integran tres puntos (cfri in/ro texto). 
Por otro lado, la forzosa asignación de tres, sicmpre tres y solamente tres puntos a cada esquema no supone precisamente una formulación ventajosa, sino la aplicación de un constructo teórico a la realidad de unos sistemas temporales cuyas unidades, creemos que con claridad más que apreciable, transmiten enfoques que no siempre, ni mucho menos, hallan su representación conveniente por medio de esqucmas dotados de tres puntos y necesitados, en consecuencia, de la aceptación de exactamente dos relaciones orientativas directas entre dichos puntos. El autor obró con un apriorismo bastante claro cuando, tras haber reconocido, correctamente a nuestro entender, la relación de simultaneidad que se establece entre el punto de referencia y el punto central $S$ en el caso del Present Perfect (cfr. supra n. 6) -identificando, en suma, la relación temporal de anterioridad a algo que es simultáneo al centro de referencias, que un siglo antes Bello $(1841: \S 39,1847: \S 638)$ había denominado ante-presente y a la que aquel mismo año Alarcos Llorach (1947: 35) hacía referencia al describir el «perfecto compuesto» como un «tiempo absoluto» modido «no como los tiempos absolutos (csto es, desde la conciencia presente), sino desde el presente gramaticals- y tras haber presentado, esta vez no en todos los casos con acierto, la existencia de una simultaneidad cntre $R$ y $E$ en el significado temporal del Simple Past (que recubre las sustancias de contenido temporal que las lenguas románicas, por ejemplo, distribuyen entre dos unidades funcionales diferentes, cfr. en castellano canté / cantaba), asertó que «We see that we need three time points even for the distinction of tenses which, in a superficial consideration, secm to concern only two time points) (1947: 289) y, acto seguido (1947: 290), aplicó el esquema de relaciones entre tres puntos a los significados temporales expresados en inglés por scis tenses (a los que se refirió respectivamente como Past Perfect, Simple Past, Present Perfect, Present, Simple Future y Future Perfect), generalizando, pues, los resultados de los análisis efectuados en un par de casos concretos a todos los demás, con, para empezar, el resultado de la redundante presentación del significado temporal del Present como $S, R, E$, esto es, como, en términos bcllistas, un co-presente, o el del Simple Future como $S, R E$, esto es, como un pos-presente ${ }^{7}$. Por otro lado, una relación temporal como la expresada en castellano por habria visto cn un cjemplo como

(1a) Supe que muy pronto habria visto por fin a Juan o en inglés por I should have seen en el caso de

(1b) T knew that I should have seen John very soon,

de ninguna manera reductible a un esquema de tres puntos, no fue en ningún momento tomada en consideración por Reichenbach (1947).

1.2. No vemos, pues, nada clara la «superioridad» que ha llegado a ser asignada a esta teoría, y hemos de señalar que algunos seguidores de esta misma idea básica han advertido la necesidad de considerar la existencia de algún punto más, habiéndose llegado a formulacioncs como las aplicadas al estudio del verbo español por Carrasco Gutiérrez (2000), quien,

7 Rcichcnbach (1947: 297) empleó los términos Simple Present y Simple Future para hacer referencia a una y otra estructuras temporalcs respcctivamentc, proponiendo el término sirnple para designar la relación de simultaneidad entre $E$ y $R$, mientras aplicaba present y future a las posiciones de $R$ en relación a $S$, respcctivamente de simultaneidad y anterioridad. 
con apoyo en Vickner (1985), ha empleado un sistema representativo de cuatro puntos ${ }^{8}$ que, aun cuando permite la representación de la relación que Bello llamó ante-pos-pretérito (cfr. supra ejs. (1 ab)), viene a complicar, en contrapartida, las cosas al imponer el mismo csquema cuádruple a todas las rclaciones temporales, con lo que, por ejemplo, las que seguimos llamando presente, pretérito o futuro, respectivamente visibles en las secuencias
(2a) en estos momentos llueve
(2b) ayer llovió
(2c) mañana lloverd́,

en que el proceso verbal no recibe sino una orientación directa desde el centro de referencias temporales, que es de simultaneidad en ( $2 a)$, de anterioridad en (2b) y de posterioridad en (2c), con el resultado de tres realizaciones de sustancia de contenido temporal a que corresponden en nuestro modelo representativo ${ }^{9}$ las formulaciones
a) $\mathrm{OOV}$
b) $\mathrm{O}-\mathrm{V}$
c) $\mathrm{O}+\mathrm{V}$,

son por la citada autora reinterpretadas como, respectivamente ${ }^{10}$,
a) $(\mathrm{R} 2, \mathrm{~F}) \mathrm{o}(\mathrm{R} 1, \mathrm{R} 2) \mathrm{o}(\mathrm{S}, \mathrm{R} 1)$
b) $(\mathrm{F}, \mathrm{R} 2) \mathrm{o}(\mathrm{R} 2, \mathrm{R} 1) \mathrm{o}(\mathrm{R} 1-\mathrm{S})$
c) (R2,F)o(R1-R2)o(S,R1),

es decir, mutatis mutandis, como las relaciones que tendríamos que llamar co-co-presente, co-co-pretérito y co-pos-presente, lo que, aparte de suponer la defensa de inexistentes relaciones de simultaneidad entre el punto $F$ y una de las referencias en los significados gramaticales expresados por formas como canté y cantaré, exige el reconocimiento de nada menos que tres orientaciones temporales relativas incluso donde no sc pueda verificar la cxistcncia de más de una por no abandonar la previa toma de postura en el sentido de que todas las significaciones temporales deban establecerse entre un número de puntos absolutamente idéntico.

Precisamente, Comrie $(1981,1985)$ tuvo el buen critcrio de no aceptar la rigidez del sistema de tres puntos (o de cualquier sistema de $n$ puntos), admitiendo que las significaciones temporales expresadas por las formas verbales se concretan en encadenamientos de orientaciones relativas, de anterioridad, simultaneidad o posterioridad, que pueden ser directamente medidas desde el centro deíctico de referencias temporalcs o bien desde alguna referencia

\footnotetext{
8 En concreto los puntos que representa mediante las abreviaturas $\mathrm{S}, \mathrm{F}, \mathrm{R} 1$ y R2, concebidos respectivamente como el momento del habla, el «punto del foco» - que «denotará cl ticmpo de la parte del evento que el aspecto hace visible» (Carrasco Gutiérrez 2000: $\$ 2.2$ ) - y dos referencias.

9 Básicamente el utilizado por Rojo (1974); cfr. Rojo \& Veiga (1999). La presentación y adaptación de este modelo a los detalles de nuestra teoría temporal puede verse en (Veiga 1991: cap. IV). Fntiéndase $O$ como abreviatura del origen o centro déctico de relaciones temporales y oV, $-\mathrm{V},+\mathrm{V}$ como simbolizaciones de, respectivamente, las orientaciones de simultaneidad, antcrioridad y posterioridad.

10 El símbolo o kindica que el significado de una forma verbal se obtiene composicionalmente) (Carrasco Gutiérrez 2000: 62).
} 
que, también directa o indirectamente, se oriente desde dicho centro deíctico. Con ello implícitamente corroboraba el fundamento del análisis efectuado por Bello tanto tiempo antes y el que Rojo (1974) había elaborado particndo, en buena medida, de corregir determinadas insuficiencias del sistema de ejes temporales de Bull (1960) ${ }^{11}$.

La necesaria toma en consideración de un número variable, teóricamente infinito, de orientaciones cncadenables como única manera de representar las efectivas posibilidades de realización de los significados temporales expresables por las formas verbales en una lengua como el castellano es aspccto básico de nuestra teoría temporal. Volviendo a considerar nuestro ejemplo de (Veiga 2004: $\$ 10.2$ [ej. 46]),

(3) Vicente dijo que Elena le habia contado que Andrés pensaba quc Ana admitiria que Luis le habia comunicado que la radio anunciaba que llovería,

ejemplo, subrayćmoslo, perfectamente gramatical, pese a su artificiosidad, en castellano, en que cada unidad verbal se orienta en correlación temporal con aquella de la que depende sintácticamente en relación directa, es decir, tomando esta última como referencia para la orientación temporal del hecho por ella expresado, exige el reconocimiento de unas cadenas de orientaciones simples de complejidad progresivamente creciente, de tal manera que cada una de las cadenas se constituye en referencia para la orientación de la relación temporal primaria (la que el proceso verbal recibe directamente desde el punto de referencia más próximo, representada en el extremo derecho de cada fórmula) expresada por la unidad verbal inmediatamente siguiente:

$\begin{array}{ll}\text { dijo: } & \mathrm{O}-\mathrm{V} \\ \text { habia contado: } & (\mathrm{O}-\mathrm{V})-\mathrm{V} \\ \text { pensaba: } & ((\mathrm{O}-\mathrm{V})-\mathrm{V}) \mathrm{oV} \\ \text { admitiria: } & ((\mathrm{O}-\mathrm{V})-\mathrm{V}) \mathrm{OV})+\mathrm{V} \\ \text { habia comunicado } & (((\mathrm{O}-\mathrm{V})-\mathrm{V}) \mathrm{oV})+\mathrm{V})-\mathrm{V} \\ \text { anunciaba: } & ((((\mathrm{O}-\mathrm{V})-\mathrm{V}) \mathrm{oV})+\mathrm{V})-\mathrm{V}) \mathrm{oV} \\ \text { lloveria: } & ((((\mathrm{O}-\mathrm{V})-\mathrm{V}) \mathrm{oV})+\mathrm{V})-\mathrm{V}) \mathrm{OV})+\mathrm{V}\end{array}$

Esto demuestra, entre otras cosas, la necesidad de diferenciar cada relación temporal concreta, contextualmente reconocible en cada caso particular, del valor funcional de cada

11 García Fernández (2004: § 2, n. 15), con todo, alinea a Comric con Reichenbach, frente a Bello, Bull, Rojo y Veiga, considerando que los sistemas propuestos por los dos primeros prevén, frente a los elaborados por los cuatro últimos, un númcro limitado de estructuras temporales. Habria que efectuar aquí muchas matizaciones, pucs el sistema de Connrie queda abierto, no menos que los de Bello o Rojo, a dar euenta de relaciones entre diversos conjuntos de puntos (aunque de hecho ninguno de estos autores llevó a sus últimas consecuencias el estudio de los posibles encadenamientos múltiples de orientaciones temporales simples); en cuanto a Bull, su sistema no dejaba de señalar unas posibilidades máximas a tenor de lo observable en la mayoría de las lenguas (cfr. supra n. 4) y, desde luego, era absolutamente rígido cn cl sentido de reducír toda posible realización de sustancia de contenido temporal a la relación entre un único punto y un único eje de referencia. Nuestra teoría temporal prevé la posibilidad, teóricamente infinita, de encadenamientos de orientaciones temporales simples, pero ello solamente en términos de sustancia, pues cada sistema configura luncionalmente una serie de parcelas integradas por todas las realizaciones de contenido temporal que compartan una serie de rasgos comunes. En cualquier caso, el establecimiento de limitaciones con pretendido alcance universal no puede efectuarse a priori sobre las restricciones de ningún sistema de representación particular. Son los sistemas los que han de adccuarse a las posibilidades de configuración lingüística de los enfoques temporales que ofrezcan las diferentes lenguas conocidas y estudiadas. 
unidad tcmporal (cfr. Veiga 1991: $\$$ IV.2.4), que puede manifcstarse en diversas realizaciones concretas de sustancia de contenido que deben ofrecer ciertas características comunes tal como los alófonos de un mismo fonema deberán realizar siempre en términos de sustancia fónica todos y cada uno de los rasgos fonológicos inherentes de la unidad funcional de que constituyen realización matcrial. Observemos que en el ejemplo anterior, en concreto, cada forma verbal de «imperfecto» (pensaba, anunciaba) expresa realizaciones de contenido temporal que ofrecen un «vector originario» (el situado más a la izquierda, en contacto directo con 0 ) de anterioridad y un «vector primario» (el situado más a la derecha) de simultaneidad, mientras que cada forma de «pluscuamperfecto» (habia contado, había comunicado) expresa rcalizaciones en que los dos vectores extremos son de anterioridad y en el caso de las formas en ría (admitiria, lloveria) el vector izquierdo es siempre negativo mientras cl situado más a la derecha es siempre positivo: he ahí las características comunes a las diversas realizaciones contextuales de unas mismas unidades temporalmente funcionales ${ }^{12}$.

Por tal motivo hemos de reiterar nuestra extrañeza ante la postura manifestada por García Fernández (1999: $\S$ 4, cfr. supra § 1.1) al considerar «superion» un modelo que impone un número preestablecido de puntos a otro que dé cuenta de la pluralidad (teóricamente infinita) de posibilidades en cuanto a encadenamientos de orientaciones temporales. Muy al contrario, todo modelo no apto para la representación de la señalada combinabilidad potencialmente infinita de las orientaciones temporales se mostrará insuficiente para simbolizar las posibilidades reales que la sustancia de contenido temporal ofrece en cuanto a realizaciones contextuales concretas en una lengua de las características del castellano, de la misma manera que, por establecer de nuevo un claro parangón con el estudio del componente fónico de la lengua, todo modelo de transcripción que limitase su conjunto de signos haciendo de entrada imposible la representación de determinados sonidos reconocibles cn el análisis fonético de una lengua dada no resultaría de aplicación a la transcripción de secuencias fónicas reales en textos emitidos en dicha lengua y su utilización implicaría la distorsión de la realidad a causa de las limitaciones del sistema de representación utilizado.

1.3. Pero la más visible limitación del modelo rcichenbachiano en lo tocante al tema concreto dcl co-pretérito, objeto de estudio en nuestro citado artículo (Veiga 2004), es su imposibilidad para diferenciar una relación temporal de anterioridad directa al centro de referencias del sistema (la relación que Bello llamó pretérito y formulamos, aprovechando en lo posible el modelo propugnado por Rojo 1974, O-V) de una relación de simultaneidad a una referencia directamente enfocada como anterior desde el mismo centro de referencias (la que Bello llamó co-pretérito y, de nuevo aprovechando el mismo modelo representativo, formulamos $(\mathrm{O}-\mathrm{V}) \mathrm{V})$. En efecto, no hay lugar para la relación de pretérito en el esquema de Reichenbach (1947), que asignaba inicialmente al Simple Past la formulación $K, E-S$ (1947: 290, cfr. supra $\S 1.1$ ), rediseñada poco más adclante por el propio autor como $E_{2} R-S$ (cfr. 1947: 297), con lo que quienes han aplicado directamente esta tcoría al estudio del verbo español aceptan dicha formulación (mutatis mutandis, la de una relación de co-pretérito, por suponer un enfoque de simultaneidad respecto de una referencia que es anterior al punto

12 Remitimos a Veiga (1991: $\S \S$ IV.2.4-5) para un análisis pormenorizado de la sustancia de contenido temporal configurada en cada una de las unidades dotadas de valor funcional en el sistema verbal español. Cfr. también la síntesis presentada en (Veiga 2004: $\$ \S 10.2-4$ ). 
central de referencias temporales) tanto para el significado temporal atribuido a la forma verbal canté como para el habitualmente atribuido a la forma verbal cantaba.

No creemos fruto de la casualidad que csta imposibilidad de distinción entre las relaciones de antcrioridad al centro de referencias y de simultaneidad a una referencia anterior a dicho centro haya surgido en una teoría concebida desde la lengua inglesa, en cuyo sistema verbal no existe una diferenciación gramatical comparable a la que las lenguas románicas establecen entre (pretéritos» y «co-pretéritos», pero hemos de reitcrar también nuestra extrañeza ante el hecho de que García Fernández (1999: $\$$ 3) se haya referido a dicha imposibilidad como «un aspecto muy interesante del modelo de Reichenbach» ${ }^{13}$, cuando no es sino una clara limitación impuesta por las rigideces de un esquema conceptual que quiere ver relaciones entre tres puntos incluso allí donde no se verifica más que una relación entrc dos (en este caso, en el enfoque temporal de pretérito, como igualmente en los de presente o futuro).

Y la inadecuación de la señalada interpretación temporal resulta ya visible en el marco de la propia teoría en que ha surgido desde el momento en que desconecta la significación temporal del Simple Past de la del Past Perfect (redenominado Anterior Past por Reichenbach 1947: 297), cuando la segunda viene a ser el resultado de la transformación de la primera a la correlación temporal con un verbo regente que exprese precisamente la relación de pretérito.

Esta crítica nos trae a la mente otra que en nuestro anterior trabajo ya hubimos de formular a paralelo problema en el sistema de represcntaciones temporales concebido por Bull (1960). Retomando nuestros ejemplos de (Veiga 2004: $\S 13.3$ [ejs. 65ab]),

$$
\mathrm{O}-\mathrm{V} \quad \text { ayer llovió }
$$

(4b) (O-V)-Vcomunicaron por la radio que el dia anterior habia llovido,

comprendemos quc, al ser (4b) el resultado de la transformación de (4a) a la correlación temporal dependiente de un pretérito, las formas del verbo llover que en ellos aparecen coinciden en expresar una misma orientación temporal primaria de anterioridad desde dos puntos de referencia distintos: directamente el centro de referencias temporales, $\mathrm{O}$, cn (4a) y una referencia $(\mathrm{O}-\mathrm{V})$, anterior a dicho punto, en (4b). Pero las formulaciones que el sistema de Bull (1960) exige para ambas unidades verbales, respectivamente
a) $\quad \operatorname{RPOV}(=$ simultaneidad a un retrospective point)
b) RP-V (= anterioridad a un retrospective point)

\footnotetext{
13 Idea que sigue suscribiendo cinco años después, al reiterar que «Un aspecto del modelo de Reichenbach que nos interesa especialmentc cs que no permite la distinción entre pretérito perfecto simple, el Pretérito de Bello, y el pretérito imperfecto, el Copretérito de Bello, en términos tcmporalesu (García Fernández 2004: 29). No es el único seguidor de esta teoría que viene a considerar positivas sus carencias. Carrasco Gutiérrez (2000: $\$ 1$ ), por ejemplo, manifiesta que cel interés de esta propuesta radica principalmente en su carácter restrictivo, es decir, en cl hecho de que proporciona un modo de limitar el númern de tiempos verbales que pueden darse en las lenguas naturales», pero un esquema con pretensiones de univcrsalidad no puede formularse sin un conocimiento suficientemente amplio, no reducido a unos pocos casos, de las organizaciones temporales presentes en los sistemas verbales de las diferentes lenguas.
} 
(cfr. Bull 1960: 72), suponen, contrariamente, la atribución a ambas de dos orientaciones distintas (de simultancidad en el primer caso y de anterioridad en el segundo) enfocadas desde un mismo punto de referencia (en ambos casos el eje retrospectivo RP).

Dc acuerdo con el sistema de representaciones de Reichenbach, la formulación aplicable a un ejemplo como (4a), o a la correspondiente construcción en inglés,

$$
\text { yesterday it rained, }
$$

vendria a ser la que el citado autor postuló para el Simple Past del inglés y, por otra parte, para el Passé Défini francés (Reichenbach 1947: 291), esto es, $R, E-S$, presentada después como $E, R-S$ (cfr. supra), formulación que no resulta coherente con la aplicada a los «pluscuamperfectos») en los correspondicntes ejemplos reconvertidos a la corrclación temporal desde un pretérito regente, tal como aparecen en (4b) o (5b),

(5b) they announced on the radio that it had rained the day before,

que no es otra que $E-R-S$, lo que pone en evidencia que, como en el caso de la representación de Bull, también la de Reichenbach impone alterar la orientación que el proceso verbal recibe desde el punto de referencia desde el que es enfocado directamente (aunque la coincidencia entre ambas representaciones no vaya mucho más allá), lo que no permitiría explicar cómo es posible que los ejemplos (4b) y (5b) resulten de las respectivas transformaciones de (4a) y (5a) a la dependencia temporal de un verbo principal en pretérito desde el momento en que dicha transformación exige la conservación de la orientación temporal primaria y la única sustitución del punto de referencia. Por supuesto, todo el error parte de atribuir a1 significado expresado por los «pretéritos» una orientación primaria de simultaneidad que hechos como el que acabamos de analizar revelan completamente inexistente.

1.4. En consecuencia, tanto en el caso de Bull como en el de los autores que han adaptado al castellano la teoría de Reichenbach admitiendo la común formulación temporal para los significados expresados por canté y cantaba, ha sido una insuficiencia teórica la responsable en último término de que uno y otros no hayan tenido más remedio que recurrir al aspecto como única explicación posible para la dilerencia de contenidos gramaticales expresada por dichas formas en igualdad de contenido modal (en combinación con el contenido a quc aludimos con la expresión Indicativo 0, cfr., p. ej., Veiga 1991: $\S \mathbb{1 1} .3 .9 .3^{14}$ ), de la misma manera que ya Alarcos Llorach (1949: $\S 13$, cfr. 1975; $§ 5,1994: \S \S 225$ ss)

14 Único valor modal que, de acuerdo con nuestra doctrina, puede expresar en castellano la forma canté, frente a la bifuncionalidad modo-temporal do cantaba (cfr. el gráfico que representa la configuración funcional de las sustancias de contenido modal y temporal en el sistema verbal español cn Veiga 1991: 219 o 1999: \$1). Aprovechemos para lamentar aquí la distorsión a que, debido a algún problema relacionado con el programa de tratamiento de textos utilizado, dicho gráfico ha sido sometido por manos que no fueron las nucstras en (Veiga 2004: $\$ 8$ ), introduciendo en él una serie de trazos horizontales que subdividen compartimentos del diseño original con el resultado de un cuadro ininteligible (1o mismo ha sucedido en la reproducción del grálico que en Veiga 2004: \$ 7.5 ilustra nuestra concepción del sistcma de oposiciones modales en el núcleo del sistema verbal español, gráflcu procedente de Veiga 1991: § II.3.9.3 - no III.2.9.3 como allí mismo so lee erróneamente, y esta vez por lapsus nucstro-, si bien en este caso solamente sobra un segmento rectilineo horizontal que parte en dos la casilla correspondiente al Subjuntivo 0). 
había postulado una oposición aspectual entre los valores que atribuía a ambas formas tras haberles señalado un mismo contenido temporal (cfr. nuestras observaciones en Veiga 1992: \$ 4.2 ó 2004: \$ 13.3).

El origen de este proceder en lo referente a la corriente reichenbachiana se halla en el propio estudio de Reichenbach (1947), quien, aun sin referencia explícita a la noción gramatical de aspecto, aludió a la diferencia existente cn francés entre los valores del Imparfait y del Passé Défini caracterizando el primero como un extended tense en contraposición al segundo (1947: 291). De esta manera, el autor citado consideraba la diferencia expresada en francés entre (6a) y (6b) como idéntica a la expresada en inglés entre (7a) y (7b), pues idéntica consideración de extended había defendido para la construcción perifrástica $I$ was seeing ${ }^{15}$ frente a I saw:
(6a) je voyais Jcan
(6b) jc vis Jean
(7a) I was seeing John
(7b) I saw John.

Parangones de este tipo cvidencian sin mayores problemas su inaceptabilidad. El mismo ejemplo de Macaulay reproducido por Reichenbach (1947: 289) para contraponer apariciones del Past Perfect a apariciones del Simple Past,

(8) [...] The Roundhead party seemed to be not merely overcome, but too much broken and scattered ever to rally again. Then commenced the reflux of public opinion. The nation began to find out to what a man it had intrusted without conditions all its dearest interests, on what a man it had lavished all its fondest affection,

muestra la correspondiente forma como necesitada en español de traducción por un «imperfecto» en el caso de seemed ( $\rightarrow$ esp. parecia) y por un «pretérito» en los de commenced y began ( $\rightarrow$ esp. comenzó, empezó), mientras que la diferencia existente en castellano entre ejemplos como (9a) y (9b) no puede establecerse en la conjugación inglesa:

(9a) aquel memo te estaba mirando todo el tiempo las piernas

(9b) aquel memo te estuvo mirando todo cl tiempo las piernas.

El simplc trasvase del valor que se quiera señalar en la construcción progresiva del inglés a los «imperfectos» románicos no conducirá sino a conclusiones tan precipitadas como equivocadas.

2. Por supuesto, la defensa del aspecto como base nocional distintiva entre los contenidos expresados por canté y cantaba (o por las correspondientes formas en otras lenguas románicas) responde tambićn a la facilidad con que ciertos matices de contenido aspectual se dejan reconocer en tantas apariciones de estas formas, mas al respecto hemos de insistir en que la funcionalidad de la distinción aspectual solamente resultaría probada en el caso de

15 En general, en sus propias palabras «In some tenses, an additional indication is given concerning the time extcnsion of the event. The English language uses the present participle to indicate that the event covers a certain stretch of timen (Rcichenbach 1947: 290). 
que se pudiera demostrar la coincidencia de ambas unidades verbales en cuanto a su valor funcional temporal; coincidencia que un análisis mínimamentc detenido revela completamente falsa, dada la simultaneidad primaria que la relación de co-pretérito comparte con la de presente y la anterioridad primaria que la de pretérito comparte con la de ante-pretérito y otras, como desde la observación de hechos reales de funcionamiento sistemático hemos mostrado, creemos que con suficiente claridad, en alguna ocasión anterior, al observar, por ejemplo, la transformación de secuencias temporalmente presentes (ejs. 10a, 11a) en los correspondicntes enfoques de co-pretérito (ejs. 10b, 11b) por medio de la correlación temporal con un verbo regente en pretérito ${ }^{16}$ :

$\begin{array}{ll}\text { (10a) dijo: llueve } & \mathrm{OoV} \\ \text { (11a) me gusta la música } & \mathrm{OoV} \\ \text { (10b) dijo que llovia } & (\mathrm{O}-\mathrm{V}) \mathrm{OV} \\ \text { (11b) me dijo que le gustaba la música } & (\mathrm{O}-\mathrm{V}) \mathrm{oV}\end{array}$

- la paralela transformación de secuencias en pretérito (ejs. 4a, 5a [cfr. supra § 1.3]) en los correspondientes ante-pretéritos (ejs. $4 \mathrm{~b}, 5 \mathrm{~b}$ [cfr. ibid.]), no estimando necesario proceder ahora a nuevas verificaciones. Hechos como estos nos mueven a destacar la validez de interpretaciones como la de Coseriu (1978: $§ 2.3 .2$, autor que insistió en la consideración de los matices aspectuales señalables en las realizaciones de contenido expresadas en Ias lenguas romances por formas del tipo canté / cantaba como cfectos secundarios de las distinciones temporales, o la de Rojo $(1974,1988,1990)$, quien concluyó que «las formas verbales españolas no tienen función aspectual, sino una simple significación secundaria y ocasional que no afecta a lo morfológicon (Rojo 1974: 143).

Nada de lo aquí expuesto debe, ni mucho menos, ser entendido en menoscabo de la legitimidad cientifica de los estudios sobre la sustancia de contenido aspectual, a que tantos autores han dedicado tantos esfuerzos. Pero el estudio sustancial de detalle, que se mostrará más o menos fructífcro y revelador de realidades concretas dependiendo de la capacidad de cada investigador, no puede por sí solo conducir a la negación de una distinción funcional basada en otra categoría gramatical diferente, categoría cuya actuación en una lengua como el castellano ha sido en tantas ocasiones mal establecida por aplicar criterios teóricos insuficientes, inadecuados o ambas cosas a la vez.

2.1. Es un hecho que el uso de canté o cantaba puede en muchas ocasiones poner de manifiesto diferencias de cnfoque aspectual. Sobran defensas de, por ejemplo, el empleo de la segunda forma como transmisora de matices de amplitud general o reitcración, también señalados en el caso de los presentes, pero dichos matices de contenido aspectual resultan, de hecho, combinables con enfoques temporales de anterioridad o posterioridad primaria $y$, así, los mismos contenidos aspectuales apreciables en (12a, 13a) o en (12b, 13b) reaparecen respectivamente en $(14 a, 15 a)$ y $(14 b, 15 b)$ :

(12a) la Tierra gira alrededor del Sol

(12b) descayuno café con lechc

16 Ejemplos que ya hemos utilizado en (Veiga 1988: $\$ 2.3$, cfr. Rojo \& Veiga 1999: $\$ 44.4$ ) y (Veiga $2004: \$$ 13.2 [ejs. 67a-b]). 
(13a) Copérnico probó que la Tierra giraba alrededor del Sol ${ }^{17}$

(13b) su abuelo desayunaba café con leche

(14a) la Tierra siempre ha girado alredcdor del Sol

(14b) durante su estancia en Madrid desayunó café con leche

(15a) la Tierra siempre gifaná alrededor del Sol

(15b) a partir de ahora desayunaré café con leche ${ }^{18}$.

Es igualmente posible la utilización de enfoques de anterioridad o posterioridad primaria para transmilir «estados permanentes». Al lado de un ejemplo como

(16) su abuelo tenía los ojos azules

son perfectamentc posibles los enfoques de pretérito y de futuro:

(17a) es lógico que mi prima tenga ojos azules; ya los tuvieron su padre y su abuelo

(17b) supongo que mis nietos también tendrán ojos azules.

El hecho de que la expresión de dichos estados por medio de canté no sea la más habitual solamente puede interpretarse en el sentido de que existen pcrspectivas temporales más fácilmente combinables con ciertos cnfoques aspectuales que con otros, cosa que nadie ha negado nunca. Las diferencias de aspecto entre los empleos más habitualcs o espontáneos no son pruebas contra la existencia de difcrenciación temporal, sino que, antes bien, la corroboran si tencmos en cuenta las características propias de una óptica temporal de simultaneidad frente a las perspectivas de anterioridad o posterioridad, fundamentalmente el hecho de que el enfoque, desde un determinado punto de referencia, de cualquier proceso o período de una cierta extensión en una perspectiva de simultancidad impone que el punto de referencia quede temporalmente englobado en la duración de dicho proceso o periodo, lo que tantas veces hallamos esgrimido a favor de la consideración aspectual imperfectiva de la significación gramatical expresada por cantaba. Tal hecho deriva directamente de la relación temporal de simultaneidad entre un punto y una entidad temporalmente cxtendida, y cualquier efecto derivado de dicha relación se observa en el caso de la realización de sustancia de contenido temporal que llamamos co-pretérito exactamente igual que en el de la que llamamos presente, que en modo alguno podríamos suponer perteneciente a una unidad temporal funcionalmente «imperfectiva» desde el momento en que no existe en castellano nada parecido a un «presente perfectivos ${ }^{19}$ que con aquella cstableciese una oposición gramatical directa de base aspectual.

17 Conocidísimo ejemplo de Beilo (1841: § 36, 1847: § 630).

18 Por supuesto, el grado de «espontaneidad» con que unos y otros enfoques temporales primarios pueden comunicar determinados matices aspectuales ne es el mismo; de ahí la presencia de ciertos indicadores temporales en los ejemplos (14ab) y (15ab). En cualquier caso, lo que nos interesa en estos momentos es poner de manifiesto la combinabilidad de estos matices con cualquiera de las tres posibles relaciones temporales primarias.

19 No lo es, desde luego, el valor de la unidad cuya realización temporal básica es la de ante-presente, expresada en Indicativo 0 por la forma he cantado, que puede enfocar, por un lado, sucesos cronológicamente situados en un pasado incluso remoto (p. ej.: «la antigïedad clásica ha sido posiblemente la época más brillante en la historia de la humanidad») o referidos a un periodo de tiempo «presente» todavía no concluido (p. ej.: «siempre me ha dado miedo la altura»). Es la conjunción de una relación temporal primaria dc anterioridad con una relación originaria de simultancidad lo que permite explicar estas y otras aplicaciones del enfoque temporal ante-presente sin necesidad alguna de recurrir a consideraciones de tipo aspectual o a la búsqueda de «dobles valores» de ningún tipo. 
2.2. Por otra parte, hemos insistido ya suficientemente, a partir de otras evidencias, contra la hipotética distintividad de un enfoque aspectual imperfectivo, durativo, extensivo, cursivo... en el significado de cantaba frente al de canté, significado que cn vano buscaríamos en ejemplos como

(18c) observaron horrorizados que la bomba hacía explosión dos minutos antes de 10 previsto

(18b) Iranscurrido el tiempo reglamentario, el partido finalizaha con el resultado inicial

(18c) en 1824, en el pueblccito austriaco de Ansfelden, nacia Anton Bruckner

(18d) tras toda una trepidante vida al filo de mil y un peligros, moria en su cama sin enterarse

(cfr. Veiga 2004: $\$ \S 2,13.5$ [ejs. 4a-c y 68]), ejemplos que, recordemos, no pueden interpretarse como resultantes de una supuesta neutralización desde el momento en que no es en ellos indiferente el uso de canté o cantaba (cfr. Veiga 2004: § 13.5 y aquí infra $\$ 3.2$ ): es precisamentc la renuncia al enfoque temporal más espontáneo lo que cxplica el recurso en ciertos estilos de lengua al co-pretérito como alternativa al pretérilo ${ }^{20}$. Igualmente, la scmelfactividad en combinación con un modo de acción «puntual» es posible mediante el recurso a la óptica propia de cantaba frente al siempre más espontáneo uso do la propia de canté, como mostrábamos en nuestro ejemplo

(19) al llegar la noche, atormentado por el remordimiento, el joven disparaba su pistola por última vez poniendo así fin a su vida,

(cfr. loc. cit. [cj. 75]). Nos hallamos, en cualquier caso, ante la búsqueda de ciertos efectos estilísticamente marcados ${ }^{21}$ (cn estos términos también se ha referido Havu 2004: $\S 2.5 .1$ a un ejemplo semejante a 18d), perfectamente explicables como derivados de la aplicación a determinados hcchos temporalmente pasados de un enfoque temporal quc, como concretamente el de co-pretérito, no es habitual en los actos de comunicación ordinarios.

3. La bibliografía a favor de un valor aspectual distintivo en el contenido gramatical expresado por cantaba en contraposición al cxpresado por canté recurre constantemente a la verificación de efectivos matices de contenido y acumula series de ejemplos que revelan la transmisión de dichos maticcs, pero es un hecho que, en general, las contraposiciones aspectualistas entre «imperfectos» y «pretéritos» señalan en los primeros efectos de contenido aspectual que resultan igualmente registrables en el caso de los «presentes», lo que

20 Esto ya lo vio con claridad Coseriu al observar este tipo de usos del «imperfecto» en las lenguas románicas: «El imperfecto parece retener su propio valor y uso, y expresar algo especial; no se trata de un simple prescindir de una diferencia porquc no se necesita hacerla, o bien porque no es necesaria: si se ticnc aqui una «sustitución», ella es aquí intencionada para expresar un cierto matiz» (Coseriu 1976: 148).

21 El uso estilístico de cantaba ha sico, precisamente, destacado por Guitart (1977: 155-6) en un ejemplo como «Llegamos a Washington cl 22 por la mañana. Esa misma tarde asesinaban a Kennedy en Dallas. La noticia nos dejó anonadados», con que el autor ilustra la que considera la ucrucial function» de este elemento verbal en «single situation predicates», a saber, «not really to look at the niddle portion of an occurrence or state but to ta'k abont situations that coocurred at some point»; señaló Guitart (ibid.) que en este ejemplo conereto la forma de «imperfectom «is used stylistically to stress the relationship between two situations that took place within the same period of time, even though their duration did not coincide at any point». 
constituye toda una evidencia a favor de que tales efectos, por visibles que resulten, no pueden considerarse evidencias de un valor funcional aspectual, que en vano buscaríamos en una unidad como el presente gramatical, no oponible, recordemos, sobre ninguna base aspectual a otro presente (cfr. supra $§ 2.1$ ). Las defensas de un valor aspectual (llamémosle «imperfectivo» o de otra manera) como característica del contenido funcional expresado por cantaba frente al expresado por canté insisten en mostrarnos hechos de sustancia, pero o bien concluyen la funcionalidad de dichos hechos partiendo de análisis insuficientes o desviados de las significaciones temporales, o bien eluden, rechazan o, simplemente (y, desce ciertas perspectivas teóricas, ya de entrada) no se plantean verificar la funcionalidad de determinadas distinciones de contenido partiendo de la necesaria conmutación de significados en igualdad de circunstancias a todos los niveles con objeto de reconocer si una distinción aspectual entre unidades modal y temporalmente coincidentes puede manifestarse en un cambio en el plano del significante.

Quc cantaba transmite habitualmente ciertos matices de contenido aspectual -insistamos, no obstante, en que no siempre (cfr. supra \$ 2.2) - cs algo innegable, pero igualmente innegable es que en una lengua como el castellano cualquier fonema funcionalmente vocal, líquido o nasal (al menos) se realiza fonéticamente sonoro, no constituyendo dicha sonoridad sino un rasgo redundante desde el momento en que no puede establecer oposición con la sordez en ninguno de estos subconjuntos fonemáticos. De la presentación y análisis de la más larga colección de espectrogramas que reflejen la vibración de las cuerdas vocales en la articulación de cualquier sonido vocal, líquido o nasal solamente puede establecerse un hecho de sustancia (de sustancia fönica en estc caso), nunca un hecho de funcionalidad, pues la funcionalidad no se verifica sobre análisis de sustancia, sino mediante otro tipo de comprobaciones. Valga este símil para insistir en que la simple observación de propiedades de sustancia de contenido aspectual, por diálanas que estas resulten - lo que tampoco se cumple en todos los casos-, no puede proporcionar directamente soluciones a problemas relativos a la organización funcional de un sistema verbal.

En cuanto a ciertas conexiones entre otros matices de contenido o aspectos sintácticos que han llegado a ser esgrimidas como pruebas de supuesto valor aspectual imperfectivo por parte de una forma de «imperfecto» (alusión a empleos «modalizados», cfr, p. ej., García Fernández 2004: $\S 8$, o lo que algunos han buscado interpretar como evidencias de un valor «anafórico», cfr., p. ej., las revisiones y comentarios de García Fernández 2004: § 4, Carrasco 2004: $\$ 3.2 .2$, o el artículo de Leonetti 2004), es evidente que no pueden efectuarse sin la necesaria consideración conjunta de todos los elementos integrantes del sistema verbal, 10 que indefectiblemente llevará a verificar que ningún valor modal cn particular que se pueda señalar propiamente en estas formas deja de resultar reconocible, sin ir más lejos, en las correspondientes formas compuestas, tan poco sospechosas de «imperfectividad», y quc tras cualquier hipotética vinculación a un «antecedente» se sitúa la realidad de una relación temporal de anterioridad originaria, igualmente compartida por enfoques temporales que pueden hallar su expresión no solamente en formas simples, sino también en formas compuestas.

No podemos permitirnos aquí la revisión crítica de todas las opiniones recogidas a estos y otros efectos en la voluminosa antología de García Fernández \& Camus Bergareche (2004), pero la lectura de algunos de los trabajos en ella recogidos pone especialmente de manifiesto, creemos, el peligro de «atomismo» inherente a cualquicr estudio que, en el caso que nos ocupa, se centre en una determinada unidad verbal, que hará patentes sus conse- 
cuencias cuando el análisis se haya efectuado sin partir de la consideración del sistema en su globalidad.

\section{REFERENCIAS BIBLTOGRÁFICAS}

Acero, J. J. (1990): «Las ideas de Reichenbach acerca del tiempo verbal». En Bosque (1990: 45-75). Alarcos Llorach, E. (1947): «Perfecto simple y perfecto compuesto en español». Revista de Filologia Española 31, 108-39. Reimpr. «Perfecto simple y compuesto» en Alarcos Llorach (19803: 1349).

Alarcos Llorach, E. (1949): «Sobre la estructura del verbo español moderno». Boletín de la Biblioteca Menéndez Pelayo 15, 50-80. Reimpr. «Sobre la estructura del verbo español» en Alarcos Llorach (19803: 50-89).

Alarcos Llorach, E. (1975): «Otra vez sobre el sistema verbal español». Homenaje a la memoria de D. Antonio Rodriguez-Moñino. Madrid: Castalia, 9-26. Reimpr. en Alarcos Llorach (1980: 120-47).

Alarcos Llorach, E. (19803): Estudios de gramática funcional del español. Madrid: Gredos.

Alarcos Llorach, E. (1994): Gramática de lu lengua española. Madrid: Espasa-Calpe.

Baquero, J. M. \& J. F. Pardo (1981): «Hacia una teoría del sistema temporal del español». Folios de Lingüistica y Literatura 2/1 (6), 5-20.

Bcllo, A. (1841): Análisis ideolófica de los tiempos de la conjugacion castellana. Valparaíso: Imprenta de M. Rivadeneyra. Repr. facs. Caracas: Cromotip, 1972.

Bcllo, A. (1847): Gramática de la lengua castellana destinada al uso de los americanos. Santiago de Chile: Imprenta del Progreso. Estudio y ed. de R. Trujillo: Gramática de la lengua castellana destinada al uso de los americanos. Con las notas de Rufino José Cuervo. Madrid: Arco/Libros, 1988.

Benveniste, E. (1965): «El lenguaje y la experiencia humana». En AA.VV: Problemas del lenguaje. Buenos Aires: Ed. Sudamericana (=Diógenes 51), 3-12. Vers. fr. «Le langagc et l'experience humaine». En AA.VV. Problèmes du langage. Paris: Gallimard, 1966 (=Diogène 51), 3-13.

Bosque, I. (cd.) (1990): Tiempo y aspecto en español. Madrid: Cátedra.

Bosque, I. \& V. Demonte (dirs.) (1999): Gramática descriptiva de la tengua española. Madrid: Real Acadcmia Española / Fundación José Ortega y Gasset / Editorial Espasa Calpe S. A.

Bull, W. E. (1960): Time, Tense, and the Verb. A Study in Theoretical and Applied Linguistics, with Porticular Attention to Spanish. Berkeley: University of California Press. Rcimpr. 1971.

Carrasco Gutiérrez A. (2000): «Los dos significados de anterioridad del pretérito imperfecto». Comunicación presentada al Congreso Internacional de Lingïistica «Léxico \& Gramática» (Lugo, 2000). Publ. en Veiga \& Pérez (2001: 59-73).

Carrasco Gutićrrez, A. (2004): «Algunas explicaciones para la simultaneidad en las oraciones subordinadas sustantivas». En Garcia Fernández \& Camus Bergareche (2004: 407-80).

Comrie, B. (1981): «On Reichenbach's Approach to Tense». En Hendrick, R. A., C. S. Masek \& M. F. Miller (eds.): Papers from the Seventeenth Regional Meeting, Chicago Linguistic Society. Chicago: Chicago Linguistic Society, 24-30.

Comrie, B. (1985): Tense. Cambridge: Cambridge University Press.

Coseriu, E. (1976): Das Romanische Verbalsystem. Tübingen: Gunther Narr. Tr. esp. de C. Opazo Velásquez: El sistema verbal románico. México: Siglo Veintiuno, 1996

Coseriu, E. (1978): «Aspect verbal ou aspects verbaux? Quelques questions de théorie et de méthode». En David, R. \& R. Martin (eds.): La notion d'aspect. Metz: Université de Metz / Klincksieck, $1980,13-25$.

García Femández, L. (1998): El aspecto gramatical en la conjugación. Madrid: Arco/Libros.

García Fernández, L. (1999); «Sobre la naturaleza de la oposición entre pretérito imperfecto y pretćrito perfecto simple». Lingǘstica Española Actual, XXI/2, 169-99. 
García Fernández, L. (2004): «El pretérito imperfecto: repaso histórico y bibliográfico». En García Fernández \& Camus Bergareche (2004: 13-95).

García Femández, L. \& B. Camus Bergareche (2004): El pretérito imperfecto. Madrid: Gredos.

Guitart, J. M. (1977): «Aspects of Spanish Aspect. A New Look at the Preterit/Imperfect Distinction». En Suñer (1977: 132-68).

Havu, J. (2004): «La accionalidad verbal y el imperfecto». En García Fernández \& Camus Bergareche (2004: 229-69).

Leonetti, M. (2004): «Por qué el imperfocto es anafórico». En García Fernández \& Camus Bergareche (2004: 481-507).

Rcichenbach, H. (1947): Elements of Symbolic Logic. New York: The Free Press / London: CollierMacMillan.

Rojo, G. (1974): 〈La temporalidad verbal en español». Verba 1, 68-149.

Rojo, G. (1988): «Temporalidad y aspecto en el verbo español». Lingüistica Española Actual 10, 195-216.

Rojo, G. (1990): «Relaciones entre temporalidad y aspecto en español». En Bosquc (1990: 17-41).

Rojo, G. \& A. Veiga (1999): «El tiempo verbal. Los tiempos simples». En Bosque \& Demonte (1999: vol. 2, 2867-2934).

Ruipérez, M. S. (1954): Estmuctura del sistema de aspectos y tiempos del verbo griego antigue. Análisis funcional sincrónico. Salamanca: Colegio Trilingüe de la Universidad (del C.S.I.C.).

Suñer, M. (ed.) (1977): Contemporary Studies in Romance Linguistics. Washington: Georgetown University School of Languages and Linguistics.

Veiga, $\Lambda$. (1988): «Planteamientos básicos para un análisis funcional de las categorías verbales en españolm. Comunicación presentada al III Coloquio Internacional de Hispanistas (Leipzig, 1988). Publ. en Wotjak \& Veiga (1990: 237-57). Reimpr. en Veiga (2002: 57-75).

Veiga, A. (1991): Condicionales, concesivas y modo verbal en español. Santiago de Compostela: Universidade de Santiago de Compostela, 1992.

Veiga, A. (1992): «La no independencia funcional del aspecto en el sistema verbal español». Español Actual 57, 65-80. Reimpr. en Veiga (2002: 103-17).

Veiga, A. (1999): «La ordenación jerárquica de las oposiciones temporales en el verbo español». Verba 26, 129-163. Reimpr. en Veiga (2002: 209-39).

Veiga, A. (2002): Esludios de morfosintaxis verbal española. Lugo: Tris Tram.

Veiga, A. (2004): «La forma verbal cantaba y la estructura modo-temporal del sistema verbal español». En García Fernández \& Camus Bergareche (2004: 96-193).

Veiga, A. \& M. R. Pérez (eds.) (2001): Lengua española y estruchuras gramaticales. Santiago de Compostela: Universidade de Santiago de Compostela.

Vickner, S. (1985): «Reichenbach Revisitcd: Onc, Two or Three Temporal Relations?». Acta Linguisfica Hafniensia 19/2: 81-98. 